\title{
Tunable White-Light-Emitting Mn-Doped ZnSe Nanocrystals
}

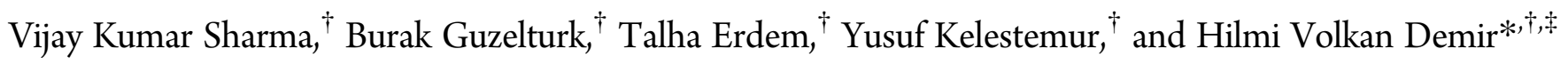 \\ ${ }^{\dagger}$ UNAM-Institute of Materials Science and Nanotechnology, Department of Electrical and Electronics Engineering, and Department \\ of Physics, Bilkent University, Ankara 06800, Turkey \\ ${ }^{\ddagger}$ Luminous! Center of Excellence for Semiconductor Lighting and Displays, School of Electrical and Electronic Engineering, and \\ School of Mathematical and Physical Sciences, Nanyang Technological University, Singapore 639798, Singapore
}

\section{Supporting Information}

ABSTRACT: We report white-light-emitting Mn-doped ZnSe nanocrystals (NCs) that are synthesized using modified nucleation doping strategy. Tailoring three distinct emission mechanisms in these NCs, which are MnSe-related blue emission (410 and $435 \mathrm{~nm}$ ), Zn-related defect state green emission $(520 \mathrm{~nm})$, and $\mathrm{Mn}$-dopant related orange emission $(580 \mathrm{~nm})$, allowed us to achieve excitation wavelength tailorable white-light generation as studied by steady state and time-resolved fluorescence spectroscopy. These NCs will be promising as single component white-light engines for
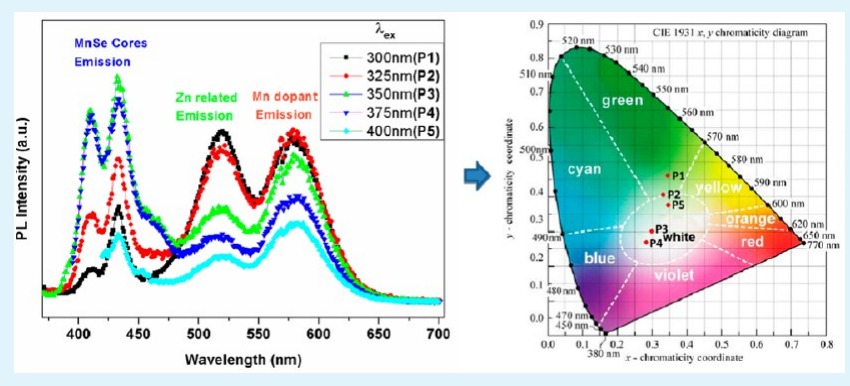
solid-state lighting.

KEYWORDS: doped nanocrystals, white-light emission, transmission electron microscopy, time-resolved photoluminescence, color properties

\section{INTRODUCTION}

White-light emission (WLE) from nanocrystals $(\mathrm{NCs})^{1}$ is presently a research area of intense interest, especially for the purpose of generation of photometrically high quality white light while maintaining the energy efficiency for solid-state lighting. One of the most common ways to generate whiteemitting NC-based phosphors is to combine red-, green-, and blue-emitting NCs in an appropriate ratio. ${ }^{1,2}$ However, when one simply mixes together the NCs of different color emission to generate white light, these systems suffer from various drawbacks including self-absorption by the small-bandgap NCs, scattering of the light at the boundaries of color conversion layers, and undesired nonradiative energy transfer among the nanoemitters. ${ }^{3}$ This also contributes to undesirable changes in the chromaticity coordinates and photometric performance of the light-emitting diodes (LEDs) due to the different relative temporal stabilities of the components of the white-LEDs. ${ }^{4}$ Current research on white-light emission has heavily focused on cadmium based NCs (e.g., by mixing red, green, and blue light from core NCs such as $\mathrm{CdS}, \mathrm{CdSe}, \mathrm{CdTe}$, or core/shell NCs including $\mathrm{CdSe} / \mathrm{ZnS}$ and $\mathrm{CdSe} / \mathrm{CdS})^{5-7}$ and magic-sized CdSe NCs. ${ }^{8}$ Recently, Rosson et al. ${ }^{9}$ reported a bright white-light emission from ultrasmall CdSe nanocrystals treated with carboxylic acids. However, due to the intrinsic toxicity of cadmium, the long-term practical applications of Cd-based NCs have been forecasted to be limited. ${ }^{10}$ Therefore, singlecomponent white-light generating NCs are highly desirable and gaining increasingly more importance in the recent years. These single component white light emitters could address the issues that have been mentioned above, along with the potential to improve the color stability, to reduce the device complexity and to lower the cost as compared to utilizing multi-NC-based color conversion layers for white-LEDs. To this end, Mn-doped NCs (e.g., $\mathrm{ZnSe},{ }^{11-13} \mathrm{ZnS},{ }^{14,15} \mathrm{CdS},{ }^{16}$ etc.) are considered to be potential candidates as single white-light generating phosphors avoiding the issues of toxicity and self-absorption. Mn-doped ZnSe NCs were already reported as white-light generating nanophosphors. For the means of achieving WLE, tuning of Mn-dopant concentration along with the wide bandedge emission from the semiconductor host was utilized, surface modification (i.e., surface-chelating) was employed to create blue-green emitting surface states, co-doping of the NCs with other dopants such as $\mathrm{Cu}$ was proposed, ${ }^{4}$ and hybridization with organic molecules was demonstrated. ${ }^{17}$ However, in all of the previous reports, the tunability of WLE from the Mn-doped NCs has not been possible, and the temporal stability of the WLE states was poor and depended on environmental conditions. ${ }^{4,11}$ In this paper, we report the generation of WLE from Mn-doped $\mathrm{ZnSe}$ NCs that is achieved via utilization of three distinct emission states in these NCs, which are here shown to be tunable as controlled during the synthesis of these NCs. These three distinct emission states are MnSe-related blue emission at 410 and $435 \mathrm{~nm}$, Zn-related green emission at $520 \mathrm{~nm}$, and Mn-dopant-related orange emission at $580 \mathrm{~nm}$. Furthermore, we have shown that, by employing these distinct states that have different photo-

Received: December 26, 2013

Accepted: February 6, 2014

Published: February 6, 2014 

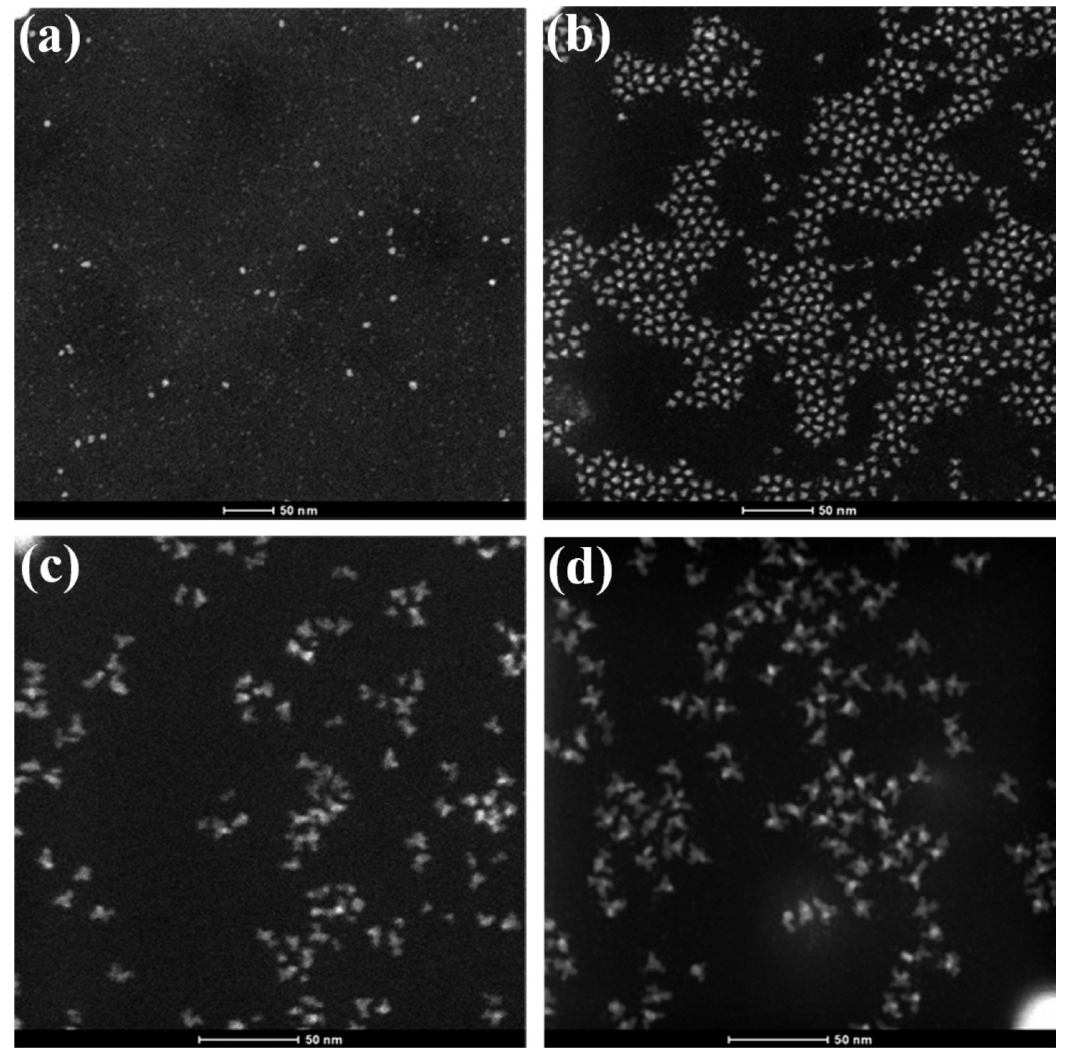

Figure 1. HAADF-STEM images of (a) MnSe cores and Mn-doped ZnSe NCs obtained after the (b) first, (c) second, and (d) third injections of Zn precursor. The scale bars are $50 \mathrm{~nm}$.

luminescence excitation spectra, WLE can be tailored by changing the wavelength of the excitation source. Here, we show that, by tuning the excitation wavelength from 325 to 400 $\mathrm{nm}$, different shades of white light can be reproducibly generated, which is technologically promising for solid-state lighting. To the best of our knowledge, this type of tunable WLE from monoemitter Mn-doped ZnSe NCs has not been reported earlier.

\section{EXPERIMENTAL SECTION}

Mn-doped ZnSe NCs are synthesized using nucleation doping strategy ${ }^{10}$ with slight modifications to obtain white light, and the NCs are purified using a new methodology as described in detail in the Supporting Information. UV-vis spectra were obtained using a UVvis spectrophotometer (Varian - Cary 100). Photoluminescence (PL) spectra (both excitation and emission) of the NCs were obtained with a fluorescence spectrophotometer (Varian - Cary Eclipse). The quantum yield of the NCs was measured using Horiba Jobin Yvon Time resolved fluorescence setup using an integrating sphere F-3018. The time-resolved fluorescence measurements were taken with a Pico Quant Fluo Time 200 setup using an excitation wavelength of $375 \mathrm{~nm}$. HAADF-STEM and HR-transmission electron microscopy (TEM Tecnai G2 F30) images were obtained using TEM operating at 300 $\mathrm{kV}$. Elemental analysis was carried out using energy dispersive X-ray spectroscopy (EDS).

\section{RESULTS AND DISCUSSION}

Transmission electron microscopy (TEM) was performed to understand the morphology and crystal structure of the Mndoped ZnSe NCs. Figure 1 shows high-angle annular dark-field (HAADF) scanning transmission electron microscope (STEM) images of Mn-doped ZnSe NCs obtained after the first, second, and third injections of $\mathrm{Zn}$ precursor on the $\mathrm{MnSe}$ cores. Figure 1a shows HAADF-STEM images of MnSe cores with two different sizes of $\sim 2$ and $4 \mathrm{~nm}$, both having a spherical geometry. Almost $90 \%$ of the NCs are of small size (i.e., $2 \mathrm{~nm}$ ), and the rest are bigger NCs (i.e., $4 \mathrm{~nm}$ ) as observed from the zoomed in image of MnSe cores, as shown in Figure S1 of the Supporting Information. Figure $1 \mathrm{~b}$ shows Mn-doped ZnSe NCs obtained after first injection of $\mathrm{Zn}$ precursor, exhibiting an average size $\sim 4.8 \mathrm{~nm}$ with a nearly spherical (i.e., tetrapod with very short branches) shape. As the number of $\mathrm{Zn}$ precursor injections is increased, the shape of the NCs deviates from nearly spherical to branched. The branched Mn-doped $\mathrm{ZnSe}$ $\mathrm{NCs}$ obtained after the third injection of $\mathrm{Zn}$ precursor have a uniform size of $\sim 9.5 \mathrm{~nm}$, with a core diameter of $\sim 4.3 \mathrm{~nm}$ and a branch length of $\sim 5.2 \mathrm{~nm}$. The number of NCs with a tetrapod shape is almost $95 \%$; there are also some bipods, as seen in Figure 1c. There are few reports on the shape control of Mn-doped ZnSe NCs. ${ }^{18,19}$ Peng et al. ${ }^{19}$ synthesized both branched and spherical Mn-doped ZnSe NCs by tuning the ratio of fatty acids and fatty amines. They observed that both the branches and the core crystallize in the zinc blende structure. We also carried out a detailed HR-TEM investigation to understand the growth mechanism of our branched Mndoped ZnSe NCs. The HR-TEM images of Mn-doped ZnSe NCs obtained after the (a) first (nearly spherical NCs) and (b) third (branched NCs) injections of Zn precursor are shown in Figure S2 of Supporting Information. For nearly spherical Mndoped $\mathrm{ZnSe}$ NCs, the interplanar spacing obtained is $\sim 3.26 \AA$ (Figure S2a), which corresponds to the (111) plane of zinc blende (ZB) structure and lies between $\sim 3.36 \AA$ (MnSe cores) (JCPDS 73-1742) and $3.24 \AA$ (ZnSe) (JCPDS 80-0021). The value is closer to that of the $\mathrm{ZnSe} \mathrm{ZB}$ structure because of the thicker $\mathrm{ZnSe}$ shell over $\mathrm{MnSe}$ core. These results were further 

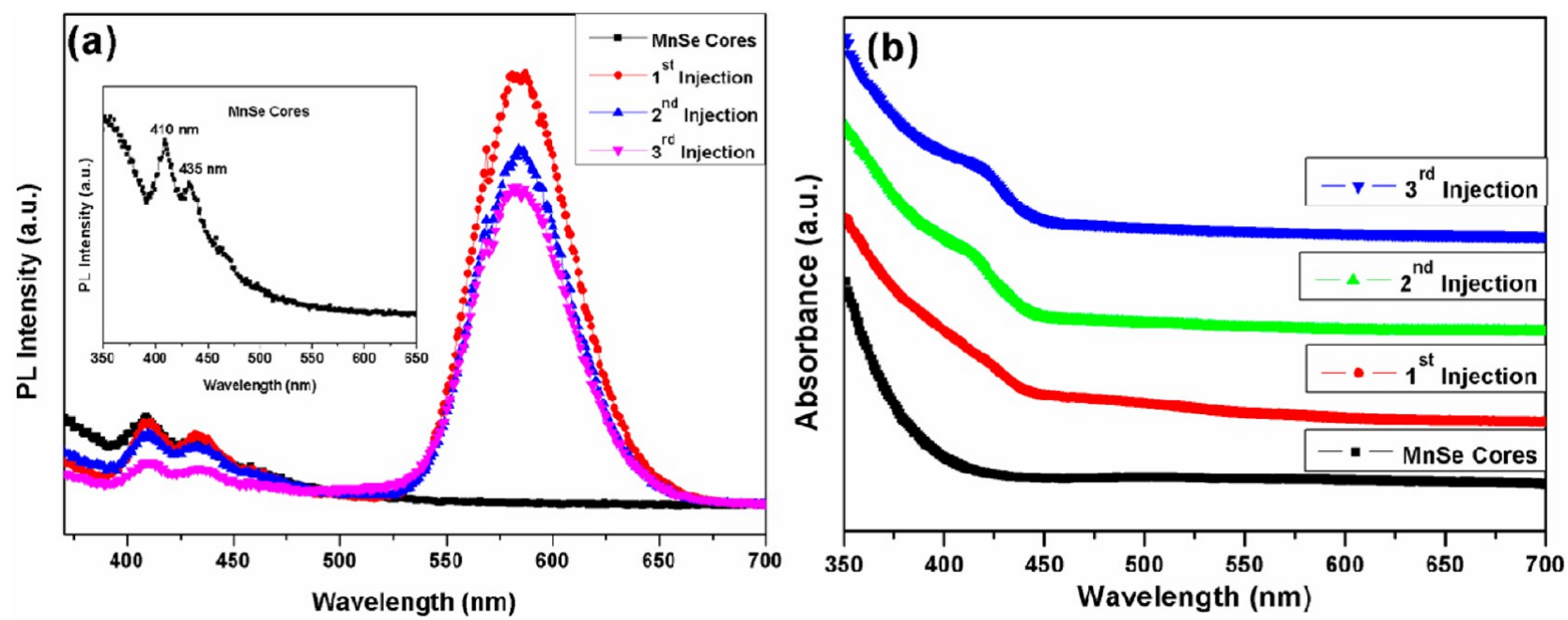

Figure 2. PL (a) and absorption spectra (b) of MnSe cores and Mn-doped ZnSe NCs obtained after first, second, and third injections of $\mathrm{Zn}$ precursor. In the inset of part a, the zoomed in PL spectra of MnSe cores are shown for clarity. PL spectra are recorded at an excitation wavelength of $300 \mathrm{~nm}$ for all cases.

verified by energy dispersive X-ray spectroscopy (EDS). For the branched Mn-doped ZnSe NCs, the interplanar $d$ spacing obtained from the branches is $\sim 3.49 \AA$ (Figure S2b). This value corresponds to the (100) plane of wurtzite $\mathrm{ZnSe}$ having a standard value $\sim 3.44 \AA$ (JCPDS 80-0008). Therefore, in our case, we observe that the core crystallizes in the zinc blende structure, whereas the branches crystallize in the wurtzite structure. Furthermore, we observed the transformation from nearly spherical to branch NCs after the second injection of $\mathrm{Zn}$ precursor on the MnSe core, with no variation in stearic acid concentration, which is in contrast to ref 19. Recently, $\mathrm{Wu}$ and Warner $^{18}$ reported shape control of Mn-doped ZnSe NCs by varying $\mathrm{Zn}$ precursor and the temperature of the injection (i.e., $\mathrm{ZnSt}_{2} / 290{ }^{\circ} \mathrm{C} /$ branched $\mathrm{NCs}$ and $\mathrm{ZnCl}_{2} / 220{ }^{\circ} \mathrm{C} /$ spherical NCs). But the maximum photoluminescence quantum efficiency reported for the branched NCs remained only at $1 \%$; in contrast, here we report an efficiency of $13.6 \%$ for the branched Mn-doped ZnSe NCs. The detailed elemental analysis done by using EDS also supports the growth mechanism revealed by HR-TEM data. The elemental concentration obtained for the spherical NCs is $\mathrm{Mn}=1.39$ atom $\% ; \mathrm{Zn}=56.22$ atom $\%$; and $\mathrm{Se}=42.37$ atom $\%$, whereas for the branched NCs the values are $\mathrm{Mn}=1.20$ atom \%; $\mathrm{Zn}=$ 66.36 atom \%; and $\mathrm{Se}=32.43$ atom \%. As the number of $\mathrm{Zn}$ precursor injections is increased, the $\mathrm{Zn}$ content is enhanced in the NCs as expected. Using EDS, Mn was detected only in the core area of the branched Mn-doped ZnSe NCs. The HR-TEM and elemental analysis of Mn-doped ZnSe NCs suggested that, in our case, the proposed atomic model for the branched $\mathrm{Mn}$ doped $\mathrm{ZnSe}$ NCs structure consists of zinc blende (111) plane of $\mathrm{ZnSe}$ core jointly with wurtzite (100) plane of $\mathrm{ZnSe}$ arms, $\mathrm{Mn}$ atoms being embedded in the core only. ${ }^{20}$

The photoluminescence (PL) spectra of $\mathrm{MnSe}$ cores and Mn-doped ZnSe NCs obtained after the first, second, and third injections of $\mathrm{Zn}$ precursor excited at $300 \mathrm{~nm}$ are shown in Figure 2a. PL spectra of Mn-doped $\mathrm{ZnSe}$ NCs were dominated by the $\mathrm{Mn}$ dopant emission at $580 \mathrm{~nm}$, accompanied with two weak emissions at 410 and $435 \mathrm{~nm}$. Mn-doped emission at 580 $\mathrm{nm}$ is independent of the shape of Mn-doped $\mathrm{ZnSe} \mathrm{NCs} \mathrm{with} \mathrm{a}$ fwhm of $52( \pm 1) \mathrm{nm}$. For the emission of the Mn dopant, we observe a decrease in the PL intensity with the increasing number of $\mathrm{Zn}$ precursor injections on the MnSe core. The decrease in the PL intensity can be accounted for by the deviation in shape from the spherical to the branched with the increasing number of $\mathrm{Zn}$ precursor injections. This change in the PL behavior is analyzed and verified with the structural features of our Mn-doped ZnSe NCs (Figure 1). These results are consistent with the previous reports. ${ }^{18,19} \mathrm{~A}$ gradual red shift in the excitonic absorption band from 410 to $420 \mathrm{~nm}$ is observed in the UV-vis absorption spectra with increasing the number of $\mathrm{Zn}$ precursor injections, as shown in Figure $2 \mathrm{~b}$. While considering the Mn-dopant emission at $580 \mathrm{~nm}$, the Stokes shift of $\sim 160-170 \mathrm{~nm}$ is achieved as compared with the first exciton absorption peak of the $\mathrm{ZnSe} \mathrm{NCs.} \mathrm{In} \mathrm{Figure} \mathrm{2a,} \mathrm{the}$ emission peaks at 410 and $435 \mathrm{~nm}$ are observed for all the samples. These blue emission peaks are attributed to the $\mathrm{MnSe}$ core of the Mn-doped $\mathrm{ZnSe}$ NCs. This is substantiated by measuring the PL spectra of the only $\mathrm{MnSe}$ cores that were used as the seed for the Mn-doped $\mathrm{ZnSe} \mathrm{NCs}$ as shown in the inset of Figure $2 \mathrm{a}$.

The UV-vis spectrum of the MnSe cores does not show any observable features at visible wavelengths up to the UV region $(350 \mathrm{~nm})$ in accordance with the previous study. ${ }^{10}$ On the basis of the UV-vis data, the optical band gap $\left(E_{\mathrm{g}}\right)$ was estimated to be approximately $3.5-3.8 \mathrm{eV}$. This value is significantly larger than that for rocksalt-type $\mathrm{MnSe}\left(E_{\mathrm{g}} \sim 2.5 \mathrm{eV}\right)^{21}$ but is consistent with the band gap of ZB-type MnSe $\left(E_{\mathrm{g}} \sim 3.4 \mathrm{eV}\right){ }^{22}$ This observation is also supported by the HR-TEM analysis of the ZB-type MnSe core as discussed above. HR-TEM image of the $\mathrm{MnSe}$ core is shown in the inset of Figure S1 of the Supporting Information. In the PL spectrum, there are two observable emission peaks at $\sim 410$ and $435 \mathrm{~nm}$. Since the characteristic emission peaks do not belong to octahedrally and tetrahedrally coordinated $\mathrm{MnSe}{ }^{23}$ these vibronic-like emission peaks possibly originate from the defects in the $\mathrm{NC}$ cores, presumed to be caused by the metastability of $\mathrm{MnSe}$ in the zinc blende structure. The exact origins of these emissions are, however, not clear. Similar emissions were also reported by Zhu et $\mathrm{al}^{24}$ from the $\mathrm{ZB}$-type $\mathrm{MnSe}$ cores while studying $\mathrm{MnSe}$ / $\mathrm{CdSe}$ core shell nanocrystals.

In our Mn-doped ZnSe NCs PL spectra (Figure 2a), we observe emissions both from Mn-dopant and $\mathrm{MnSe}$ cores, 

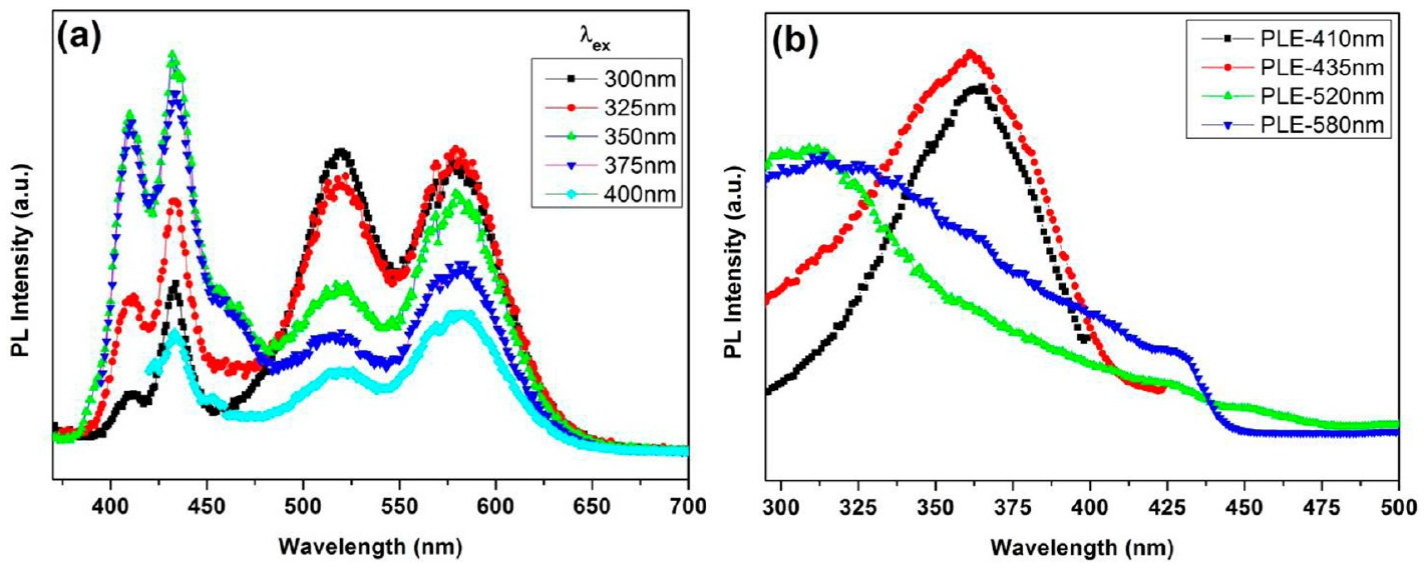

Figure 3. (a) PL spectra of the modified Mn-doped ZnSe NCs under excitation at various wavelengths. (b) PLE spectra of all emissions in Mndoped ZnSe NCs.

simultaneously. These emissions cannot be explained by nucleation doping strategy alone. In our case, on the basis of optical and structural characterization, there are two possible reasons for these emissions. First, there is a possibility that, rather than a nucleation doping synthesis, in our case we have a two-step nucleation synthesis. In a two-step nucleation model, the amorphous polynuclei or polymolecules aggregate together in the first step, and in the second step, these amorphous structures start to adjust the positions of atoms and reorganize these clusters into the crystalline form. ${ }^{25}$ If two regions on the surface crystallize at the same time in the second step of surface nucleation, then they could not fuse into one single crystal and a twin boundary is formed. We also observed twinning in our synthesized Mn-doped ZnSe NCs as shown in Figure S3 of Supporting Information. For a second possibility, in our synthesis we found $\mathrm{MnSe}$ cores of two sizes with $90 \%$ of the $\mathrm{MnSe}$ cores being smaller $(2 \mathrm{~nm})$ and the remaining being larger $(4 \mathrm{~nm})$. By coating these NCs with $\mathrm{ZnSe}$, the smaller and bigger MnSe cores both lead to Mn-doped ZnSe NCs but with thicker and thinner $\mathrm{ZnSe}$ shells, respectively. For the thinner shells there are emissions from $\mathrm{MnSe}$ cores, too. This hypothesis is also supported by the PL spectra in Figure 2a; as the $\mathrm{ZnSe}$ coating on $\mathrm{MnSe}$ core increases, the intensity of emission from MnSe cores decreases. PL spectra of Mn-doped ZnSe NCs normalized at $580 \mathrm{~nm}$ also support our hypothesis as shown in the Figure S4 of Supporting Information. Also, it is observed that as we increase the concentration of $\mathrm{Mn}$ in the synthesis the intensity of the PL emission (410 and $435 \mathrm{~nm}$ ) from the MnSe cores increases, which is not shown here. Therefore, in our case we have emissions both from Mn-dopant and $\mathrm{MnSe}$ cores simultaneously for the Mn-doped ZnSe NCs.

In this study, we further utilize $\mathrm{Mn}$-doped $\mathrm{ZnSe} \mathrm{NCs}$ as monoemitter WLE agents. In addition to the blue emission from $\mathrm{MnSe}$ cores and the orange emission from Mn-dopant, a green emission was required to achieve high-quality white light. By tuning the zinc stearate to stearic acid ratio simultaneously with the $\mathrm{Zn}$ to Se ratio, we were able to obtain the green emission in addition to the blue and orange emission using a modified recipe (hereafter called as modified synthesis, described in detail in the Experimental Section). Thus, the white light generation was realized from the modified synthesis of Mn-doped $\mathrm{ZnSe} \mathrm{NCs}$ as a result of the combination of light emission in orange (Mn-dopant emission), in green ( $\mathrm{Zn}$ related defects), and in blue (MnSe cores emission) simultaneously.
These three emissions can be controlled to achieve high-quality white light. The modified Mn-doped ZnSe NCs demonstrated excitation wavelength dependent emission color tunability, which was studied at room-temperature as shown in Figure 3a. The HAADF-STEM images of these NCs obtained using the modified synthesis exhibits tetrapode shape with an average size $\sim 8.5 \mathrm{~nm}$ as shown in Figure S5 of the Supporting Information.

We observe that PL spectra obtained from the modified synthesis of Mn-doped ZnSe NCs shows four emission peaks; two blue emission peaks at 410 and $435 \mathrm{~nm}$, one green emission peak at $520 \mathrm{~nm}$, and one orange emission peak at 580 $\mathrm{nm}$. The blue emission peaks at 410 and $435 \mathrm{~nm}$ are the emissions from $\mathrm{MnSe}$ cores as discussed above. The green emission around $520 \mathrm{~nm}$ is attributed to "self-activated" luminescence, as a consequence of donor-acceptor pairs that are related to $\mathrm{Zn}$ vacancy and crystal defects (interstitial states or associated with dislocations, stacking faults, and nonstoichiometric defects). ${ }^{26,27}$ The green band is observed in the samples annealed in $\mathrm{Zn}$ vapor, vacuum, or reducing atmosphere, resulting in $\mathrm{Zn}$ excess. ${ }^{28}$ Green emission was also reported in both undoped and $\mathrm{Mn}$ doped $\mathrm{ZnS}$ nonorods by Biswas et al., ${ }^{29}$ and they concluded that this emission is due to zinc-vacancy related defect states. Our samples were grown in $\mathrm{Zn}$-rich conditions; thus, we believe that the strong emission may be ascribed to the interstitial $\mathrm{Zn}$ defect and nonstoichiometric defects. The orange emission at $580 \mathrm{~nm}$ is due to the ${ }^{4} \mathrm{~T}_{1}-{ }^{6} \mathrm{~A}_{1}$ transition of the Mn impurity excited by energy transfer from the $\mathrm{ZnSe}$ lattice.

To investigate the origin of these emission bands, PLE spectra of Mn-doped $\mathrm{ZnSe} \mathrm{NCs}$ were recorded. Figure $3 \mathrm{~b}$ shows the PLE spectra for the emission at 410, 435, 520, and $580 \mathrm{~nm}$. The PLE peak position for the $580 \mathrm{~nm}$ emission wavelength is $310 \mathrm{~nm}$, indicating that the orange emission is from Mn-doped ZnSe NCs. ${ }^{10}$ PLE spectra of the orange emission have a bump at $430 \mathrm{~nm}$, which is similar to the first exciton peak in the absorption spectra of Mn-doped ZnSe NCs shown in Figure S6 of Supporting Information. We observed that in this case the absorption peak is $10 \mathrm{~nm}$ red-shifted in comparison to the branched one due to thicker $\mathrm{ZnSe}$ coating. In contrast, the PLE spectrum recorded for the $520 \mathrm{~nm}$ emission exhibits a maxima at $310 \mathrm{~nm}$ along with a shoulder bumping at $360 \mathrm{~nm}$. The $360 \mathrm{~nm}$ PLE peak is associated with Zn-related defects as reported previously. ${ }^{30}$ The green emission observed at $520 \mathrm{~nm}$, which is new in our case, is attributed to 

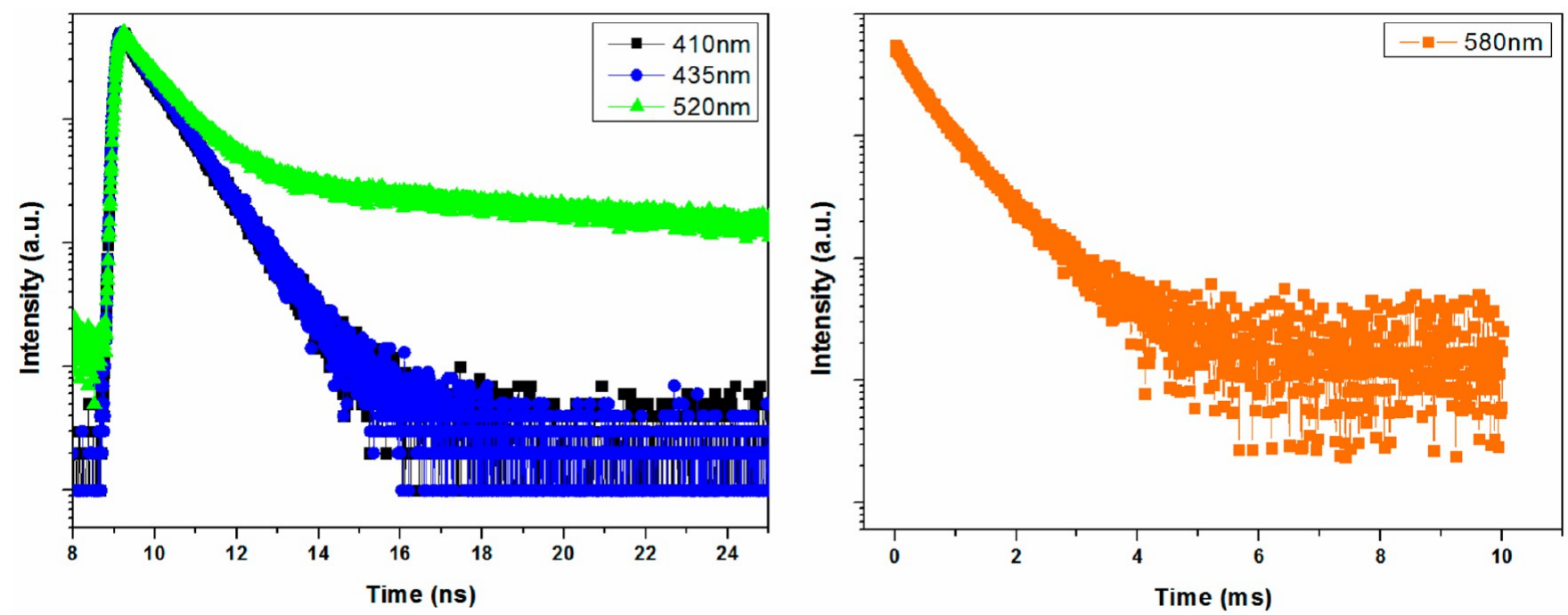

Figure 4. TRPL spectroscopy of Mn-doped ZnSe NCs.

the modified synthesis of these NCs. Thus, the green emission is due to zinc related defects (excess Zinc) in the Mn-doped ZnSe NCs. ${ }^{28}$ The green emission $(520 \mathrm{~nm})$ has a maximum at $310 \mathrm{~nm}$, but immediately afterward the emission decreases with increasing wavelength. Thus, the green emission is not related to $\mathrm{Mn}$-doping in $\mathrm{ZnSe}$. Also, in Figure $3 \mathrm{~b}$, we have recorded PLE plots for 410 and $435 \mathrm{~nm}$, both having their maximum at $365 \mathrm{~nm}$. After $330 \mathrm{~nm}$ the blue emission (410 and $435 \mathrm{~nm}$ ) starts to increase and reaches its maximum at $365 \mathrm{~nm}$. Thus, PLE results indicate that all these emissions have different origins which are further confirmed via time-resolved photoluminescence (TRPL) spectroscopy.

Figure 4 shows the fluorescence decay curves for all four (410, 435, 520, and $580 \mathrm{~nm}$ ) emissions of Mn-doped $\mathrm{ZnSe}$ NCs excited at $375 \mathrm{~nm}$. The PL decay profile of the orange emission $(580 \mathrm{~nm})$ is found to be single exponential with an average lifetime of $0.59 \mathrm{~ms}$. Such a slow decay further confirmed the attribution of this emission band to the spin forbidden doped $\mathrm{Mn}^{2+}{ }^{4} \mathrm{~T}_{1}$ to ${ }^{6} \mathrm{~A}_{1}$ transition. Then, we studied the emission kinetics for the blue emission at wavelengths of 410 and $435 \mathrm{~nm}$, which exhibited a single exponential decay with fast emission decay lifetimes of 0.61 and $0.63 \mathrm{~ns}$, respectively. Generally for semiconductor NCs, the fluorescence lifetimes are of the order of a couple of nanoseconds or longer; thus, these fast decay kinetics for the blue emission at 410 and $435 \mathrm{~nm}$ (arising from MnSe cores emission) is due to trap state emission of the MnSe cores together with possible competition with other nonradiative channels such that overall PL decay lifetime is quite short, or the other explanation to these vibronic-like blue emissions would be the formation of very small $\mathrm{MnSe}$ nanoclusters. Having similar lifetimes for 410 and $435 \mathrm{~nm}$ indicates that there exists excitons which are highly bound to each other (similar to Frenkel excitons rather than Wannier-Mott excitons) since the MnSe core is too small (like a nanocluster) before the $\mathrm{ZnSe}$ shell growth. Also, these two emission states were observed from the steady state PL of only $\mathrm{MnSe}$ cores (Figure 2b). Also, relatively good PL QY of these blue emission states supports the fact that nonradiative decay channels cannot be highly dominant. Moreover, PLE of these states (see Figure 3b) has a resonance feature around $365 \mathrm{~nm}$ that supports the hypothesis that the blue emission is more likely to be arising from the MnSe nanoclusters rather than highly inefficient trap states.

For the green emission at $520 \mathrm{~nm}$, the average lifetime of $1.72 \mathrm{~ns}$ is obtained, which is attributed to zinc related defects introduced in our system by the modified synthesis. The value $1.72 \mathrm{~ns}$ is the amplitude weighted average lifetime of the 520 $\mathrm{nm}$ emission obtained after fitting the curve with three exponentials (reduced $\chi^{2} \sim 1$ ). These lifetime components are $0.804 \mathrm{~ns}(92.96 \%), 3.74 \mathrm{~ns}$ (3.37\%), and $23.02 \mathrm{~ns}(3.67 \%)$. The fastest lifetime component is attributed to the direct recombination lifetime of the $520 \mathrm{~nm}$ emission state. Longer lifetime components may be related to the shallow trap state mediated emission causing a delayed emission with longer fluorescence lifetime components. ${ }^{31}$ There is a possibility that, since the sample has excess $\mathrm{Zn}$, the cleaning process may produce some oxidized $\mathrm{Zn}$, which may be a reason for the green emission in PL. ${ }^{32}$ The lifetime of the green emission is slower than the blue emission and much faster than the orange emission suggesting that these three emission states are distinct and have different emission mechanisms that are independently controlled as shown by PLE spectra in Figure $3 \mathrm{~b}$. Therefore, the above results indicate that, by controlling the synthesis parameters, we can tune the WLE from the Mn-doped $\mathrm{ZnSe}$ NCs, thus making it possible to be used as versatile monoemitters for WLE. The intensity of these relative emissions can be tuned by varying the excitation wavelengths; thus, white light generation can be easily controlled and tuned.

Figure 5 shows the chromaticity coordinates of the emission from modified Mn-doped ZnSe NCs excited at $300 \mathrm{~nm}$ (P1), $325 \mathrm{~nm}$ (P2), $350 \mathrm{~nm}$ (P3), $375 \mathrm{~nm}$ (P4), and $400 \mathrm{~nm}$ (P5), where these different excitation wavelengths lead to chromaticity coordinates of $(0.34,0.46),(0.33,0.40),(0.30,0.30)$, $(0.28,0.26)$, and $(0.34,0.37)$, respectively. The photometric color properties (LER, CCT, CRI) are calculated for all excitation wavelengths and are listed in Table 1 . Here the chromaticity coordinates corresponding to the excitation wavelengths $325,350,375$, and $400 \mathrm{~nm}$ fall within the white region of the $1931 \mathrm{CIE}$ (Commission Internationale de L'Eclairaged) diagram ${ }^{33}$ (Figure 5). Through excitation source tuning, white light from the NCs can be tuned to its different shades with luminous efficacy up to $400 \mathrm{~lm} / \mathrm{W}$ and correlated color temperature in the range of $5000-11000 \mathrm{~K}$. Therefore, 


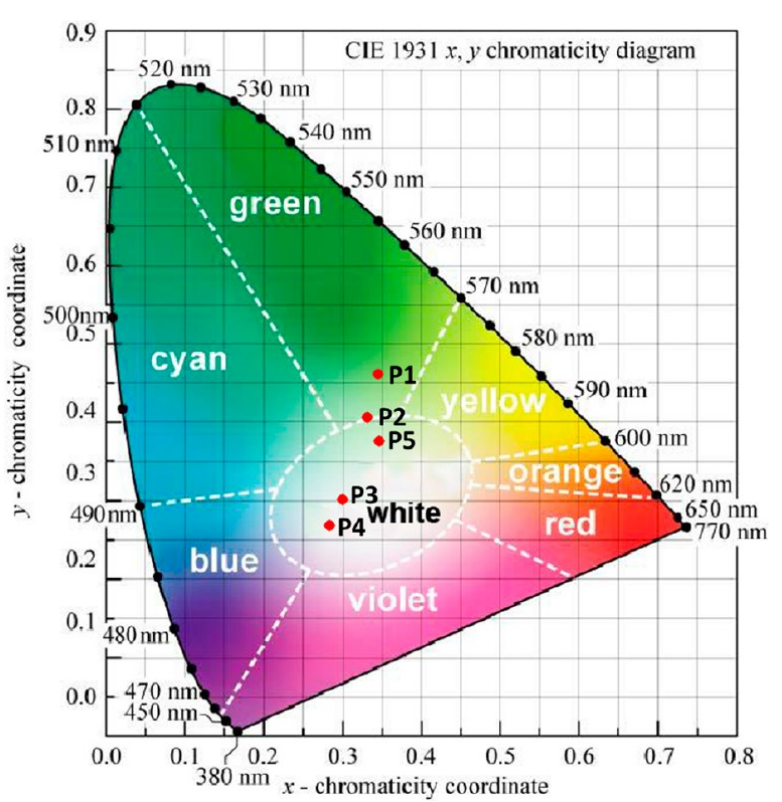

Figure 5. CIE chromaticity diagram for Mn-doped ZnSe NCs.

Table 1. Color Properties of Mn-Doped ZnSe NCs

\begin{tabular}{lcccc}
$\lambda_{\text {ex }}(\mathrm{nm})$ & $(x, y)$ & LER $\left(\mathrm{lm} / \mathrm{W}_{\text {opt }}\right)$ & CCT $(\mathrm{K})$ & CRI \\
$300(\mathrm{P} 1)$ & $(0.3456,0.4615)$ & 401 & 5213 & 48 \\
$325(\mathrm{P} 2)$ & $(0.3311,0.4057)$ & 354 & 5556 & 51 \\
$350(\mathrm{P} 3)$ & $(0.2993,0.3010)$ & 265 & 7769 & 60 \\
$375(\mathrm{P} 4)$ & $(0.2834,0.2677)$ & 236 & 11170 & 64 \\
$400(\mathrm{P} 5)$ & $(0.3458,0.3758)$ & 357 & 5049 & 50 \\
\hline
\end{tabular}

WLE with high color quality is successfully achieved for Mndoped ZnSe NCs.

\section{CONCLUSIONS}

In this paper, we report WLE from the Mn-doped ZnSe NCs that are synthesized with a modified synthesis reported here. As a result, we achieve three controllable distinct emission states from these NCs; blue emission from the MnSe cores, orange emission from the Mn dopant, and green emission from the Zn-related defect states. All these emissions are reproducibly controlled by small variation in the synthesis parameters. The tailoring of the white emission via excitation source wavelength owing to the distinct PLE characteristics of the three emission states enabled us to tune the white light from the same NCs for the first time in the literature. These Mn-doped $\mathrm{ZnSe}$ NCs are promising as versatile light emitting materials suitable for various applications ranging from solid-state lighting to bioimaging.

\section{ASSOCIATED CONTENT}

\section{S Supporting Information}

HAADF-STEM and HR-TEM images of MnSe cores (Figure S1); HR-TEM images of Mn-doped ZnSe NCs obtained after the (a) first and (b) third injections of $\mathrm{Zn}$ precursor (Figure S2); HR-TEM image of Mn-doped ZnSe NCs showing twin boundary (Figure S3); PL spectra of Mn-doped ZnSe NCs obtained after the first, second, and third injections of $\mathrm{Zn}$ precursor normalized at $580 \mathrm{~nm}$ (Figure S4); HAADF-STEM image of the Mn-doped $\mathrm{ZnSe} \mathrm{NCs}$ that generates white light (Figure S5); absorption spectra of Mn-doped ZnSe NCs that generates white light (Figure S6). This material is available free of charge via the Internet at http://pubs.acs.org.

\section{AUTHOR INFORMATION}

\section{Corresponding Author}

*(H.V.D.) E-mail: volkan@bilkent.edu.tr. Tel.:+90312-2901021.

\section{Notes}

The authors declare no competing financial interest.

\section{ACKNOWLEDGMENTS}

This work is supported by EU-FP7 Nanophotonics4Energy NoE and TUBITAK EEEAG, 110E217. H.V.D. gratefully acknowledges ESF-EURYI and TUBA-GEBIP. T.E. and Y.K. acknowledge TUBITAK fellowship.

\section{REFERENCES}

(1) Erdem, T.; Demir, H. V. Color science of nanocrystal quantum dots for lighting and displays. Nanophotonics 2013, 2, 57-81.

(2) Ali, M.; Chattopadhyay, S.; Nag, A.; Kumar, A.; Sapra, S.; Chakraborty, S.; Sarma, D. D. White-light emission from a blend of CdSeS nanocrystals of different Se:S ratio. Nanotechnology 2007, 18, 075401.

(3) Roushan, M.; Zhang, X.; Li, J. Solution-Processable White-LightEmitting Hybrid Semiconductor Bulk Materials with High Photoluminescence Quantum Efficiency. Angew. Chem. 2012, 124, 451-454.

(4) Panda, S. K.; Hickey, S. G.; Demir, H. V.; Eychmuller, A. Bright White-Light Emitting Manganese and Copper Co-Doped ZnSe Quantum Dots. Angew. Chem., Int. Ed. 2011, 50, 4432-4436.

(5) Lita, A.; Washington, A. L., II; van de Burgt, L.; Strouse, G. F.; Stiegman, A. E. Stable Efficient Solid-State White-Light-Emitting Phosphor with a High Scotopic/Photopic Ratio Fabricated from Fused CdSe-Silica Nanocomposites. Adv. Mater. 2010, 22, 3987-3991.

(6) Lee, J.; Sundar, V. C.; Heine, J. R.; Bawendi, M. G.; Jensen, K. F. Full Color Emission from II-VI Semiconductor Quantum DotPolymer Composites. Adv. Mater. 2000, 12, 1102-1105.

(7) Demir, H. V.; Nizamoglu, S.; Erdem, T.; Mutlugun, E.; Gaponik, N.; Eychmuller, A. Quantum dot integrated LEDs using photonic and excitonic color conversion. Nano Today 2011, 6, 632-647.

(8) Bowers, M. J., II; McBride, J. R.; Rosenthal, S. J. White-Light Emission from Magic-Sized Cadmium Selenide Nanocrystals. J. Am. Chem. Soc. 2005, 127, 15378-15379.

(9) Rosson, T. E.; Claiborne, S. M.; McBride, J. R.; Stratton, B. S.; Rosenthal, S. J. Bright White Light Emission from Ultrasmall Cadmium Selenide Nanocrystals. J. Am. Chem. Soc. 2012, 134, 8006-8009.

(10) Pradhan, N.; Peng, X. Efficient and Color-Tunable Mn-Doped $\mathrm{ZnSe}$ Nanocrystal Emitters: Control of Optical Performance via Greener Synthetic Chemistry. J. Am. Chem. Soc. 2007, 129, 33393347.

(11) Shao, P.; Wang, H.; Zhang, Q.; Li, Y. White light emission from Mn-doped ZnSe d-dots synthesized continuously in microfluidic reactors. J. Mater. Chem. 2011, 21, 17972-17977.

(12) Lee, S. M.; Hwang, C. S. Synthesis of a White-Light-Emitting ZnSe:Mn Nanocrystal via Thermal Decomposition Reaction of Organometallic Precursors. Bull. Korean Chem. Soc. 2013, 34, 321324.

(13) Yang, B.; Zhang, J.; Cui, Y.; Wang, K. White light-emitting diode coated with $\mathrm{ZnSe}: \mathrm{Mn} / \mathrm{ZnSe}$ nanocrystal films enveloped by $\mathrm{SiO}_{2}$. Appl. Opt. 2011, 50, G137-G141.

(14) Quan, Z.; Yang, D.; Li, C.; Kong, D.; Yang, P.; Cheng, Z.; Lin, J. Multicolor Tuning of Manganese-Doped ZnS Colloidal Nanocrystals. Langmuir 2009, 25, 10259-10262.

(15) Kar, S.; Biswas, S. White Light Emission from Surface-Oxidized Manganese-Doped ZnS Nanorods. J. Phys. Chem. C 2008, 112, 11144-11149. 
(16) Nag, A.; Sarma, D. D. White Light from $\mathrm{Mn}^{2+}$-Doped CdS Nanocrystals: A New Approach. J. Phys. Chem. C 2007, 111, 1364113644.

(17) Wu, P.; Yan, X. P. Doped quantum dots for chemo/biosensing and bioimaging. Chem. Soc. Rev. 2013, 42, 5489-5521.

(18) Wu, Y. A.; Warner, J. H. Shape and property control of Mn doped ZnSe quantum dots: from branched to spherical. J. Mater. Chem. 2012, 22, 417-424.

(19) Viswanatha, R.; Battaglia, D. M.; Curtis, M. E.; Mishima, T. D.; Johnson, M. B.; Peng, X. Shape Control of Doped Semiconductor Nanocrystals (d-Dots). Nano Res. 2008, 1, 138-144.

(20) Manna, L.; Milliron, D. J.; Meisel, A.; Scher, E. C.; Alivisatos, A. $\mathrm{P}$. Controlled growth of tetrapod-branched inorganic nanocrystals. Nat. Mater. 2003, 2, 382-385.

(21) Kan, S.; Felner, I.; Banin, U. Synthesis, Characterization, and Magnetic Properties of $\alpha$-MnS Nanocrystals. Isr. J. Chem. 2001, 41, $55-61$.

(22) Ishibe, I.; Nabetani, Y.; Kato, T.; Matsumoto, T. MBE growth and RHEED characterization of $\mathrm{MnSe} / \mathrm{ZnSe}$ superlattices on GaAs

(100) substrates. J. Cryst. Growth 2000, 214/215, 172-177.

(23) Heimbrodt, W.; Goede, O.; Tschentscher, I.; Weinhold, V.; Klimakow, A.; Pohl, U.; Jacobs, K.; Hoffmann, N. Optical study of octahedrally and tetrahedrally coordinated MnSe. Physica B 1993, 185, $357-361$.

(24) Zhu, K.; Zhang, X.; Wang, L.; Zhang, H.; Wei, J.; Wang, L.; Zhou, M.; Feng, B. Manganese-doped MnSe/CdSe core/shell nanocrystals: Preparation, characterization, and study of growth mechanism. J. Mater. Res. 2011, 26, 2400-2406.

(25) Bryan, J. D.; Gamelin, D. R. Doped Semiconductor Nanocrystals: Synthesis, Characterization, Physical Properties, and Applications. Prog. Inorg. Chem. 2005, 54, 47-126.

(26) Bukaluk, A.; Trzcinski, A.; Firszt, F.; Legowski, S.; Meczynska, $\mathrm{H}$. Auger depth profile analysis and photoluminescence investigations of $\mathrm{Zn}_{1-\mathrm{x}} \mathrm{Mg}_{\mathrm{x}} \mathrm{Se}$ alloys. Surf. Sci. 2002, 507-510, 175-180.

(27) Geng, B. Y.; You, J. H.; Zhan, F. M.; Kong, M. G.; Fang, C. H. Controllable Morphology Evolution and Photoluminescence of $\mathrm{ZnSe}$ Hollow Microspheres. J. Phys. Chem. C 2008, 112, 11301-11306.

(28) Liu, M.; Kitai, A. H.; Mascher, P. Point defects and luminescence centres in zinc oxide and zinc oxide doped with manganese. J. Lumin. 1992, 54, 35-42.

(29) Biswas, S.; Kar, S.; Chaudhuri, S. Optical and Magnetic Properties of Manganese-Incorporated Zinc Sulfide Nanorods Synthesized by a Solvothermal Process. J. Phys. Chem. B 2005, 109, $17526-17530$

(30) Kar, S.; Biswas, S. White Light Emission from Surface-Oxidized Manganese-Doped ZnS Nanorods. J. Phys. Chem. C 2008, 112, 11144-11149.

(31) Kloepfer, J. A.; Bradforth, S. E.; Nadeau, J. L. Photophysical Properties of Biologically Compatible CdSe Quantum Dot Structures. J. Phys. Chem. B 2005, 109, 9996-10003.

(32) Zhong, Y.; Djurisic, A. B.; Hsu, Y. F.; Wong, K. S.; Brauer, G.; Ling, C. C.; Chan, W. K. Exceptionally Long Exciton Photoluminescence Lifetime in $\mathrm{ZnO}$ Tetrapods. J. Phys. Chem. C 2008, $112,16286-16295$.

(33) Schubert, E. F. Light-Emitting Diodes, 2nd ed.; Cambridge University Press: Cambridge, U.K., 2006. 